\title{
Atomic-Resolution Imaging of Graphene Using an Ultrahigh-vacuum Microscope with a High-brightness Electron Gun
}

Hidetaka Sawada $^{1}$, Shigeyuki Morishita ${ }^{1}$, Yuji Kohno ${ }^{1}$, Takeo Sasaki ${ }^{2}$, Masaki Mukai ${ }^{1}$, Yung-Chang Lin $^{3}$, Ryosuke Senga ${ }^{3}$ and Kazu Suenaga ${ }^{3}$

${ }^{1}$ JEOL Ltd., Akishima, Tokyo, Japan, ${ }^{2}$ JEOL Ltd., Welwyn Garden City, England, United Kingdom, ${ }^{3}$ AIST, Tsukuba, Ibaraki, Japan

During TEM/STEM observation or EELS/EDS analysis in electron microscopes, reduction of specimen damage is important in maintaining the structure of two-dimensional materials. To reduce the knock-on damage, we are operating instruments with low energy electrons below $60 \mathrm{keV}$ in TEM and STEM [1]. On the other hand, it is reported specimen etching by an electron beam with residual gases in a microscope column; the etching also causes structure change of the specimen [2]. To suppress such a specimen etching, higher vacuum environment is required in the microscope column. Thus, we have developed an ultrahigh-vacuum low-voltage scanning transmission electron microscope [3]. The instrument is the third microscope developed in Triple C project (Triple C \#3). Triple C project was supported by JST under the Research Acceleration Program (2012-2016).

Improvement in resolution is another target of development in Triple C \#3 microscope. To improve the resolution at low accelerating voltages, we installed a delta corrector for an illumination system, which is a 5th-order geometrical aberration corrector [4]. The sixth-order three-lobe aberration becomes a dominant residual aberration, which limits the aberration corrected semi-angle to around $70 \mathrm{mrad}$ [5]. The delta corrector allows atomic-resolution STEM imaging at accelerating voltages of less than $60 \mathrm{kV}$. In addition to the resolution, probe current is important for analyses such as atomic resolution EELS mapping. In this study, we have developed a new cold field emission gun (CFEG) with high brightness to produce a small probe with a high beam current. The high-brightness CFEG was installed in the ultrahigh-vacuum lowvoltage microscope (Triple C \#3).

The STEM performance of the developed microscope was evaluated by using a specimen of monolayer graphene. Figure 1 shows annular-dark-field (ADF) STEM images of graphene at $60 \mathrm{kV}$ in several conditions by changing the current of the electron probe at the specimen plane. Figure 1a shows an image with a probe current of $54 \mathrm{pA}$. The C-C dumbbells with a separation of $0.142 \mathrm{~nm}$ are clearly resolved. The Fourier transform of the image shows spots corresponding to $12 \mathrm{~nm}^{-1}=(0.08 \mathrm{~nm})^{-1}$, as shown in Fig. 1 b. Figure $1 \mathrm{c}$ was taken with a shorter dwell time of $16 \mu \mathrm{s} / \mathrm{px}$. The total acquisition time of the image is about $4 \mathrm{~s}$. We can detect carbon atomic positions on a monolayer graphene in this relatively fast scan image due to high-brightness CFEG. It indicates that the possibility to improve the time resolution of the STEM imaging of two-dimensional materials composed of light elements. Figure $1 \mathrm{~d}$ shows an image with a large probe current of $273 \mathrm{pA}$. Even the images of each atom are slightly larger than those in Fig. 1a, we can distinguish each carbon atom with such a high current. The small probe with high current produced by the developed CFEG will be useful for atomic resolution EELS mapping.

During these observations, the pressure in the specimen chamber was less than $5 \times 10^{-7} \mathrm{~Pa}$. Even that we continuously observed the monolayer graphene without largely changing the position (within around 20 $\mathrm{nm}$ ) to obtain these STEM images, the specimen was not damaged owing to small amount of the residual gases, which typically introduced damage related to etching. The low-voltage STEM with higher vacuum is useful for atomic resolution analyses of various sensitive two-dimensional materials. 

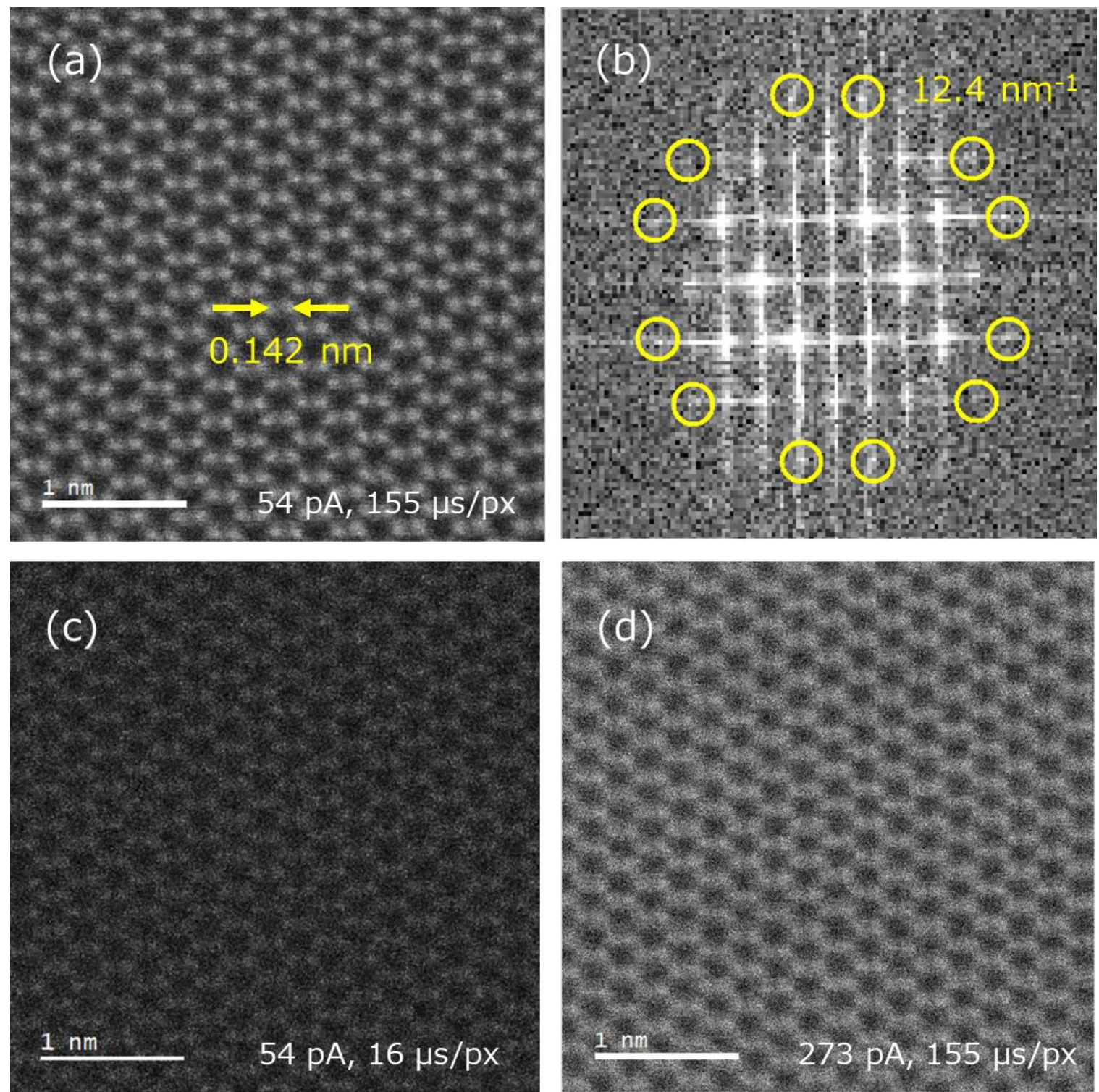

Figure 1. (a) Raw ADF STEM image of a monolayer graphene (512 x 512 pixels). Convergence semiangle is $40 \mathrm{mrad}$. A probe current and a dwell time are $54 \mathrm{pA}$ and $155 \mu \mathrm{s} / \mathrm{px}$, respectively. (b) Fourier transform of the image (a). (c) Graphene image with a dwell time of $16 \mu \mathrm{s} / \mathrm{px}$. Total acquisition time is around 4s. (c) Graphene image with a probe current of $273 \mathrm{pA}$.

References [1] K. Suenaga and M. Koshino, Nature 468 (2010) 1088-1090. [2] G. T. Leuthner, et al., Ultramicroscopy 203 (2019) 76-81. [3] T. Sasaki, et al., Microsc. Microanal. 23 Suppl 1 (2017) 468-469. [4] H. Sawada, et al., J. Electron Microsc. 58 (2009) 341-347. [5] S. Morishita, et al., Microscopy 67 (2018) 156-163. 